\title{
Effects of Risk Aversion on Market Outcomes: A Stochastic Two-Stage Equilibrium Model
}

Kazempour, Jalal; Pinson, Pierre

Published in:

Proceedings of International Conference on Probabilistic Methods Applied to Power Systems 2016

Link to article, DOI:

10.1109/PMAPS.2016.7764200

Publication date:

2016

Document Version

Peer reviewed version

Link back to DTU Orbit

Citation (APA):

Kazempour, J., \& Pinson, P. (2016). Effects of Risk Aversion on Market Outcomes: A Stochastic Two-Stage Equilibrium Model. In Proceedings of International Conference on Probabilistic Methods Applied to Power Systems 2016 IEEE. https://doi.org/10.1109/PMAPS.2016.7764200

\section{General rights}

Copyright and moral rights for the publications made accessible in the public portal are retained by the authors and/or other copyright owners and it is a condition of accessing publications that users recognise and abide by the legal requirements associated with these rights.

- Users may download and print one copy of any publication from the public portal for the purpose of private study or research.

- You may not further distribute the material or use it for any profit-making activity or commercial gain

- You may freely distribute the URL identifying the publication in the public portal 


\title{
Effects of Risk Aversion on Market Outcomes: A Stochastic Two-Stage Equilibrium Model
}

\author{
S. Jalal Kazempour and Pierre Pinson \\ Department of Electrical Engineering \\ Technical University of Denmark, Lyngby, Denmark \\ seykaz@elektro.dtu.dk; ppin@elektro.dtu.dk
}

\begin{abstract}
This paper evaluates how different risk preferences of electricity producers alter the market-clearing outcomes. Toward this goal, we propose a stochastic equilibrium model for electricity markets with two settlements, i.e., day-ahead and balancing, in which a number of conventional and stochastic renewable (e.g., wind power) producers compete. We assume that all producers are price-taking and can be risk-averse, while loads are inelastic to price. Renewable power production is the only source of uncertainty considered. The risk of profit variability of each producer is incorporated into the model using the conditional value-at-risk $(\mathrm{CVaR})$ metric. The proposed equilibrium model consists of several risk-constrained profit maximization problems (one per producer), several curtailment cost minimization problems (one per load), and power balance constraints. Each optimization problem is then replaced by its optimality conditions, resulting in a mixed complementarity problem. Numerical results from a case study based on the IEEE one-area reliability test system are derived and discussed.
\end{abstract}

Index Terms-Risk, equilibrium, wind power, uncertainty, dayahead, balancing.

\section{NOTATION}

Indices and Sets:

$l \quad$ Index for loads

$g \quad$ Index for conventional producers

$q \quad$ Index for renewable power producers

$\omega \quad$ Index for renewable power scenarios

\section{Constants:}

$C_{g} \quad$ Offer price of conventional producer $g[€ / \mathrm{MWh}$, equal to its marginal cost

$C_{g}^{\mathrm{DW}}$ Offer price of conventional producer $g$ for downward reserve deployment [€/MWh]

$C_{g}^{\mathrm{UP}} \quad$ Offer price of conventional producer $g$ for upward reserve deployment [€/MWh]

$P_{l} \quad$ Power consumption of load $l$ [MW]

$P_{g}^{\max }$ Capacity of conventional producer $g$ [MW]

$R_{g}^{\mathrm{DW}}$ Maximum downward reserve limit of conventional producer $g[\mathrm{MW} / \mathrm{h}]$

$R_{g}^{\mathrm{UP}} \quad$ Maximum upward reserve limit of conventional producer $g[\mathrm{MW} / \mathrm{h}]$

$V_{l} \quad$ Value of curtailed load for load $l$ [€/MWh]

$W_{q, \omega}$ Renewable power realization of producer $q$ under scenario $\omega[\mathrm{MW}]$
$W_{q}^{\max }$ Installed capacity of renewable power producer $q$ [MW]

$\varphi_{\omega} \quad$ Probability of scenario $\omega$

$\alpha \in(0,1)$, which is confidence level used to compute the conditional value-at-risk

$\beta \quad$ A non-negative weighting parameter modeling the tradeoff between expected profit and conditional value-at-risk

Variables in Day-Ahead Stage:

$p_{g}^{\text {DA }} \quad$ Dispatched power output of conventional producer $g$ [MW]

$w_{q}^{\mathrm{DA}} \quad$ Dispatched power output of renewable power producer $q[\mathrm{MW}]$

$\lambda^{\mathrm{DA}} \quad$ Day-ahead market-clearing price [€/MWh]

Variables in Balancing Stage:

$p_{l, \omega}^{\text {cur }}$ Involuntarily load curtailment of load $l$ under scenario $\omega[\mathrm{MW}]$

$r_{g, \omega}^{\mathrm{DW}}$ Downward reserve provided by conventional producer $g$ under scenario $\omega$ [MW]

$r_{g, \omega}^{\mathrm{UP}} \quad$ Upward reserve provided by conventional producer $g$ under scenario $\omega[\mathrm{MW}]$

$w_{q, \omega}^{\mathrm{B}} \quad$ Renewable power deviation of producer $q$ under scenario $\omega[\mathrm{MW}]$

$\lambda_{\omega}^{\mathrm{B}} \quad$ Probability-weighted balancing market-clearing price under scenario $\omega[€ / \mathrm{MWh}]$

Risk Variables:

$\zeta \quad$ Value-at-risk

$\eta \quad$ Auxiliary variable to compute the conditional valueat-risk

\section{INTRODUCTION}

The continuously increasing penetration of variable renewable energy sources, e.g., wind and solar power producers, in electricity markets motivates to use stochastic platforms for various decision-making problems, e.g., market clearing. For example, references [1]-[4] address the market-clearing 
problem using a stochastic setup for perfectly competitive electricity markets with two settlements: day-ahead and balancing. In these works, all producers are assumed to be price-taking and risk-neutral, and a market operator clears the market by solving a single optimization problem, whose objective is to maximize the expected social welfare of the market, or to minimize the expected system cost in markets with inelastic loads. The market-clearing outcomes are in fact the solution of an equilibrium problem, in which no producer desires to deviate from its power schedule. In other words, none of producers can increase its expected profit in the equilibrium point by changing unilaterally its schedule.

In electricity markets with stochastic renewable energy sources, all producers, either renewable or conventional, are exposed to the risk of profit variability. This raises several technical questions: how to model different risk preferences of producers? What are their impacts on market-clearing outcomes? and etc. Note that the market-clearing outcomes (equilibrium solution) should support all risk preferences, otherwise the unsatisfied producers may move the equilibrium point to their own favor.

Several papers incorporate the risk management into the market-clearing problem. Reference [5] proposes a stochastic two-settlement equilibrium model for markets considering renewable incentives, e.g., feed-in premiums, that generate risk exposures for both conventional and renewable producers. A distributed market-clearing mechanism is proposed in [6] to minimize the system cost, including conventional generation costs, end-user disutility, as well as a risk measure of the system redispatching cost. In addition, a risk-based day-ahead unit commitment model is proposed in [7] that considers the risks of the loss of load, wind curtailment and transmission congestion caused by wind power uncertainty.

In a similar line with [5], we propose a stochastic two-stage equilibrium model, in which all conventional and renewable producers are price-taking and can be risk-averse, while loads are inelastic to price. Renewable power production is the only source of uncertainty considered. The risk of profit variability of each producer is incorporated into the model using the conditional value-at-risk (CVaR) metric [8]. Inspired by [9] that proposes a single-stage deterministic equilibrium model, the stochastic two-stage equilibrium model proposed in the current paper consists of several risk-constrained profit maximization problems (one per producer), several curtailment cost minimization problems (one per load), and power balance constraints. Each optimization problem is then replaced by its optimality conditions, resulting in a mixed complementarity problem. The numerical results achieved in this paper show how market-clearing prices, system cost, and power dispatches vary with different risk preferences of producers.

\section{Proposed Equilibrium Model}

The proposed stochastic two-stage equilibrium model consists of four blocks as illustrated in Fig. 1. In the first block, each conventional producer $g$, either risk-neutral or risk-averse, maximizes its expected profit in day-ahead and balancing stages. Similarly, each renewable power producer

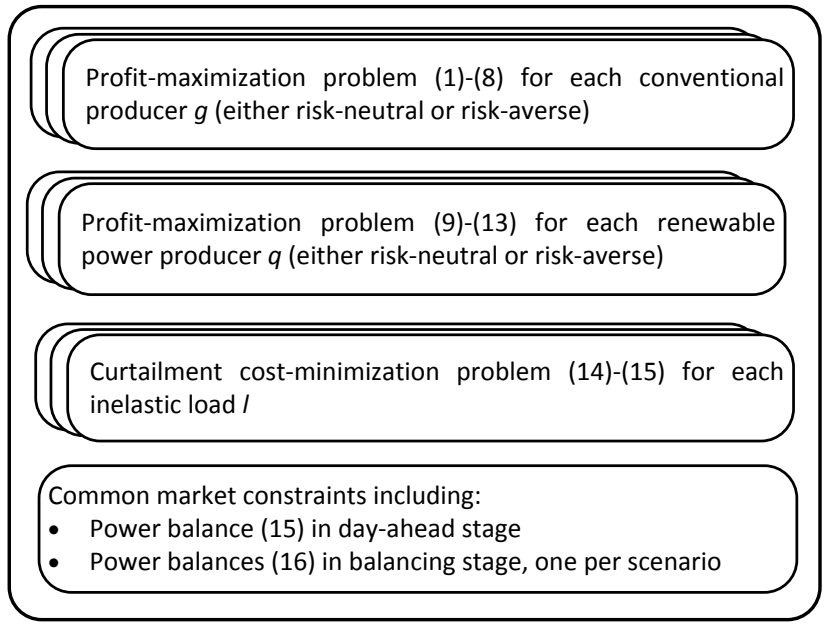

Fig. 1. Structure of the proposed stochastic two-stage equilibrium model with risk-averse producers

$q$, either risk-neutral or risk-averse, maximizes its profit in the second block. In addition, each inelastic load minimizes its cost incurred by involuntarily load curtailment in the third block. As common constraints of the market included in the fourth block, we enforce the power balances in day-ahead and balancing stages, whose dual variables provide the marketclearing prices. Note that those prices are variables in the equilibrium model, while they are treated as fixed values (parameters) within the producers' and loads' optimization problems, i.e., blocks 1 to 3 .

Problem (1)-(8) below presents the profit-maximization problem of each conventional producer $g$. Note that the dual variables are indicated within constraints following a colon.

$$
\begin{aligned}
& \left\{\underset{p_{g}^{\mathrm{DA}}, r_{g, \omega}^{\mathrm{UP}}, r_{g, \omega}^{\mathrm{DW}}, \zeta_{g}, \eta_{g, \omega}}{\operatorname{arime}} p_{g}^{\mathrm{DA}}\left(\lambda^{\mathrm{DA}}-C_{g}\right)\right. \\
& +\sum_{\omega} \varphi_{\omega}\left[\frac{\lambda_{\omega}^{\mathrm{B}}}{\varphi_{\omega}}\left(r_{g, \omega}^{\mathrm{UP}}-r_{g, \omega}^{\mathrm{DW}}\right)-C_{g}^{\mathrm{UP}} r_{g, \omega}^{\mathrm{UP}}+C_{g}^{\mathrm{DW}} r_{g, \omega}^{\mathrm{DW}}\right] \\
& +\beta_{g}\left[\zeta_{g}-\frac{1}{1-\alpha_{g}} \sum_{\omega} \varphi_{\omega} \eta_{g, \omega}\right]
\end{aligned}
$$

subject to:

$$
\begin{aligned}
& 0 \leq p_{g}^{\mathrm{DA}} \leq P_{g}^{\max } \quad: \underline{\mu}_{g}^{\mathrm{DA}}, \bar{\mu}_{g}^{\mathrm{DA}} \\
& p_{g}^{\mathrm{DA}}+r_{g, \omega}^{\mathrm{UP}} \leq P_{g}^{\max }: \bar{\rho}_{g, \omega}^{\mathrm{B}} \quad \forall \omega \\
& r_{g, \omega}^{\mathrm{DW}} \leq p_{g}^{\mathrm{DA}} \quad: \underline{\rho}_{g, \omega}^{\mathrm{B}} \quad \forall \omega \\
& 0 \leq r_{g, \omega}^{\mathrm{UP}} \leq R_{g}^{\mathrm{UP}} \quad: \underline{\rho}_{g, \omega}^{\mathrm{UP}}, \bar{\rho}_{g, \omega}^{\mathrm{UP}} \quad \forall \omega \\
& 0 \leq r_{g, \omega}^{\mathrm{DW}} \leq R_{g}^{\mathrm{DW}} \quad: \underline{\rho}_{g, \omega}^{\mathrm{DW}}, \bar{\rho}_{g, \omega}^{\mathrm{DW}} \quad \forall \omega \\
& \eta_{g, \omega} \geq 0 \quad: \rho_{g, \omega}^{\eta} \quad \forall \omega \\
& \zeta_{g}-\left[p_{g}^{\mathrm{DA}}\left(\lambda^{\mathrm{DA}}-C_{g}\right)+\frac{\lambda_{\omega}^{\mathrm{B}}}{\varphi_{\omega}}\left(r_{g, \omega}^{\mathrm{UP}}-r_{g, \omega}^{\mathrm{DW}}\right)\right. \\
& \left.-C_{g}^{\mathrm{UP}} r_{g, \omega}^{\mathrm{UP}}+C_{g}^{\mathrm{DW}} r_{g, \omega}^{\mathrm{DW}}\right] \leq \eta_{g, \omega}: \rho_{g, \omega}^{\mathrm{CVaR}} \forall \omega \\
& \} \forall g \text {. }
\end{aligned}
$$

The objective function (1) includes the day-ahead profit of 
producer $g$ (the first line), the expected profit of that unit in balancing stage (the second line), and the CVaR multiplied by a non-negative factor, i.e., $\beta_{g}$, making a tradeoff between the expected profit and the CVaR (the third line). Note that $\beta_{g}=0$ means that conventional producer $g$ is risk-neutral, while its non-zero value implies that producer $g$ is risk averse. A higher positive value for $\beta_{g}$ makes conventional producer $g$ more risk averse [8].

Constraint (2) enforces the lower and upper bounds for power schedule of producer $g$ in day-ahead market, and constraints (3)-(6) restrict the reserve deployment of that producer based on its generation capacity and maximum downward and upward reserve limits. Finally, (7) and (8) are the CVaR constraints. Note that $\zeta_{g}$ is a free variable and its optimal value refers to the value-at-risk (VaR), which is the largest value for the profit of producer $g$ such that the probability of the profit being lower than or equal to this value is lower than or equal to $1-\alpha_{g}$. Based on the $\mathrm{VaR}$, the $\mathrm{CVaR}$ is the expected value of profit values that are lower than or equal to the VaR.

Similarly, optimization problem (9)-(13) below allows each renewable power producer $q$ to make a trade-off between its expected profit and CVaR:

$$
\begin{aligned}
& \left\{\underset{w_{q}^{\mathrm{DA}}, w_{q, \omega}^{\mathrm{B}}, \zeta_{q}, \eta_{q, \omega}}{\operatorname{Maximize}} w_{q}^{\mathrm{DA}} \lambda^{\mathrm{DA}}+\sum_{\omega} w_{q, \omega}^{\mathrm{B}} \lambda_{\omega}^{\mathrm{B}}\right. \\
& +\beta_{q}\left[\zeta_{q}-\frac{1}{1-\alpha_{q}} \sum_{\omega} \varphi_{\omega} \eta_{q, \omega}\right]
\end{aligned}
$$

subject to:

$$
\begin{aligned}
& 0 \leq w_{q}^{\mathrm{DA}} \leq W_{q}^{\max } \quad: \underline{\mu}_{q}^{\mathrm{DA}}, \bar{\mu}_{q}^{\mathrm{DA}} \\
& \left.\begin{array}{l}
0 \leq\left(w_{q}^{\mathrm{DA}}+w_{q, \omega}^{\mathrm{B}}\right) \leq W_{q, \omega} \quad: \underline{\rho}_{q, \omega}^{\mathrm{B}}, \bar{\rho}_{q, \omega}^{\mathrm{B}} \quad \forall \omega \\
\eta_{q, \omega} \geq 0 \quad: \rho_{q, \omega}^{\eta} \quad \forall \omega \\
\zeta_{q}-\left[w_{q}^{\mathrm{DA}} \lambda^{\mathrm{DA}}+\frac{\lambda_{\omega}^{\mathrm{B}}}{\varphi_{\omega}} w_{q, \omega}^{\mathrm{B}}\right] \leq \eta_{q, \omega} \quad: \rho_{q, \omega}^{\mathrm{CVaR}} \quad \forall \omega \\
\end{array}\right\} \forall q .
\end{aligned}
$$

The objective function (9) includes the expected profit of renewable power producer $q$ (the first line) and its weighted $\mathrm{CVaR}$ (the second line). The renewable power production cost is assumed to be zero. Constraint (10) limits the day-ahead schedule of producer $q$ based on its installed capacity. For each scenario, constraint (11) restricts the total production of producer $q$ to be non-negative and lower than the power realization under that scenario. Note that this constraint allows excess renewable power to be curtailed. Finally, (12) and (13) are CVaR constraints.

In addition, each inelastic load minimizes its expected curtailment cost as given by (14)-(15) below:

$$
\begin{aligned}
& \left\{\underset{p_{l, \omega}^{\text {cur }}}{\operatorname{Minimize}} \sum_{\omega} \varphi_{\omega} p_{l, \omega}^{\text {cur }}\left(V_{l}-\frac{\lambda_{\omega}^{\mathrm{B}}}{\varphi_{\omega}}\right)\right. \\
& \text { subject to: } \\
& 0 \leq p_{l, \omega}^{\text {cur }} \leq P_{l} \quad: \underline{\rho}_{l, \omega}^{\text {cur }}, \bar{\rho}_{l, \omega}^{\text {cur }} \quad \forall \omega
\end{aligned}
$$

TABLE I

DATA FOR CONVENTIONAL PRODUCERS

\begin{tabular}{c|c|c|c|c|c|c}
\hline $\begin{array}{l}\text { Unit } \\
(g)\end{array}$ & $\begin{array}{c}P_{g}^{\max } \\
{[\mathrm{MW}]}\end{array}$ & $\begin{array}{c}R_{g}^{\mathrm{UP}} \\
{[\mathrm{MW}]}\end{array}$ & $\begin{array}{c}R_{g}^{\mathrm{DW}} \\
{[\mathrm{MW}]}\end{array}$ & $\begin{array}{c}C_{g} \\
{[€ / \mathrm{MWh}]}\end{array}$ & $\begin{array}{c}C_{g}^{\mathrm{UP}} \\
{[€ / \mathrm{MWh}]}\end{array}$ & $\begin{array}{c}C_{g}^{\mathrm{DW}} \\
{[€ / \mathrm{MWh}]}\end{array}$ \\
\hline G1 & 40 & 0 & 0 & 11.09 & - & - \\
\hline G2 & 40 & 0 & 0 & 11.09 & - & - \\
\hline G3 & 152 & 40 & 40 & 16.60 & 18.26 & 14.94 \\
\hline G4 & 152 & 40 & 40 & 16.60 & 18.26 & 14.94 \\
\hline G5 & 300 & 105 & 105 & 18.52 & 20.37 & 16.67 \\
\hline G6 & 591 & 210 & 210 & 19.10 & 21.01 & 17.19 \\
\hline G7 & 60 & 60 & 60 & 22.41 & 24.65 & 20.17 \\
\hline G8 & 155 & 30 & 30 & 14.08 & 15.49 & 12.67 \\
\hline G9 & 155 & 30 & 30 & 14.08 & 15.49 & 12.67 \\
\hline G10 & 400 & 0 & 0 & 10.17 & - & - \\
\hline G11 & 400 & 0 & 0 & 10.17 & - & - \\
\hline G12 & 300 & 0 & 0 & 0 & - & - \\
\hline G13 & 310 & 60 & 60 & 14.08 & 15.49 & 12.67 \\
\hline G14 & 350 & 40 & 40 & 12.46 & 13.71 & 11.21 \\
\hline
\end{tabular}

Finally, constraints (16) and (17) are included in the equilibrium model enforcing the power balance equalities in dayahead and balancing markets, respectively:

$$
\begin{aligned}
\sum_{l} P_{l}-\sum_{g} p_{g}^{\mathrm{DA}}- & \sum_{q} w_{q}^{\mathrm{DA}}=0 \quad: \lambda^{\mathrm{DA}} \\
\sum_{g}\left(r_{g, \omega}^{\mathrm{UP}}-r_{g, \omega}^{\mathrm{DW}}\right)+ & \sum_{q} w_{q, \omega}^{\mathrm{B}} \\
& +\sum_{l} p_{l, \omega}^{\mathrm{cur}}=0 \quad: \lambda_{\omega}^{\mathrm{B}} \quad \forall \omega .
\end{aligned}
$$

All optimization problems included in the equilibrium model (1)-(17) are continuous and linear. This allows us to replace each optimization problem by its Karush-Kuhn-Tucker (KKT) optimality conditions. Appendices A, B and C include the KKT conditions associated with optimization problems of conventional producers, renewable power producers and loads, respectively. This way, the proposed equilibrium model (1)(17) is recast as a stochastic nonlinear mixed-complementarity problem (MCP) including market constraints (16)-(17) and KKT conditions (18)-(45). The resulting MCP is solvable by PATH under GAMS or other complementarity problem solvers.

\section{NumeriCAl Results}

This section provides numerical results for a case study based on the IEEE one-area reliability test system [10] including 17 inelastic loads, 14 conventional producers (G1 to G14), and two wind power producers (Q1 and Q2). Pursuing simplicity, the network constraints are not enforced. The consumption level of each load is identical to that in [10] raised by $5 \%$. A single hour is considered, and the total load is $2992.5 \mathrm{MW}$. The technical data for conventional producers are given in Table I. The total conventional generation capacity is $3405 \mathrm{MW}$, whereas the total installed wind power capacity is $1586 \mathrm{MW}$. The wind power uncertainty is modeled through 48 equiprobable scenarios. According to the scenarios considered, the wind power penetration, i.e., total expected wind power divided by total load, is $23.3 \%$. In addition, the total standard deviation of the two wind producers is $363 \mathrm{MW}$, which equals 
TABLE II

EQUILIBRIUM RESULTS FOR DIFFERENT CASES

\begin{tabular}{|c|c|c|c|c|c|}
\hline & Case 1 & Case 2 & Case 3 & Case 4 & Case 5 \\
\hline Day-ahead market-clearing price [€/MWh] & 16.481 & 16.557 & 16.759 & 16.600 & 17.840 \\
\hline Expected balancing market-clearing price $[€ / \mathrm{MWh}]$ & 16.481 & 16.557 & 16.759 & 12.456 & 10.808 \\
\hline Total dispatch of conventional producers in day-ahead [MW] & 2234.1 & 2234.1 & 2234.1 & 2637.8 & 2534.1 \\
\hline Total dispatch of wind power producers in day-ahead [MW] & 758.4 & 758.4 & 758.4 & 354.7 & 458.4 \\
\hline Total expected curtailed wind power $[\mathrm{MW}]$ & 15.3 & 15.3 & 36.4 & 48.1 & 107.6 \\
\hline Total expected curtailed load [MW] & 0 & 0 & 0 & 0 & 0 \\
\hline Expected system cost $[€]$ including generation and load curtailment costs & 25863.0 & 25898.2 & 26056.8 & 26442.6 & 27150.7 \\
\hline Total payment of loads [€] & 49319.0 & 49546.8 & 50151.3 & 49675.5 & 53386.2 \\
\hline \multirow{3}{*}{ Wind power producer Q1 } & 4827.9 & 4856.0 & 3922.1 & 3523.8 & 5109.7 \\
\hline & -3398.3 & -3349.2 & -17652.8 & 1276.3 & -779.2 \\
\hline & 387.1 & 387.1 & 387.1 & 82.6 & 385.7 \\
\hline \multirow{3}{*}{ Wind power producer Q2 } & 4575.8 & 4601.8 & 3725.4 & 4170.1 & 2691.2 \\
\hline & -4059.7 & -4000.8 & -18209.3 & -149.0 & 732.0 \\
\hline & 371.3 & 371.3 & 371.3 & 272.1 & 72.7 \\
\hline \multirow{3}{*}{ Conventional producer G5 } & 108.7 & 77.5 & 323.3 & 333.2 & 170.1 \\
\hline & -2264.3 & 0 & 0 & -2178.1 & -13.6 \\
\hline & 105.0 & 0 & 0 & 105.0 & 20.1 \\
\hline \multirow{3}{*}{ Conventional producer G6 } & 127.1 & 127.1 & 665.8 & 602.9 & 41.6 \\
\hline & -1644.3 & -4654.4 & -47.0 & -5533.7 & 0 \\
\hline & 59.1 & 164.1 & 20.1 & 210.0 & 0 \\
\hline \multirow{3}{*}{ Conventional producer G7 } & 18.1 & 18.1 & 158.5 & 117.5 & 310.5 \\
\hline & 0 & 0 & 0 & -2526.3 & -1942.4 \\
\hline & 0 & 0 & 0 & 60.0 & 60.0 \\
\hline
\end{tabular}

to $52 \%$ of their expected production. The value of curtailed load $\left(V_{l}\right)$ for all loads are identical and equal to \$200/MWh.

We consider the following five cases to evaluate how different risk preferences of producers change the market-clearing outcomes:

- Case 1) all producers are risk-neutral, i.e., $\beta_{g}=0, \forall g$ and $\beta_{q}=0, \forall q$.

- Case 2) this case is similar to Case 1 , but $\beta_{\mathrm{G} 5}=1$. In this case, conventional producer G5 is the sole riskaverse agent of the market.

- Case 3) this case is similar to Case 1 , but $\beta_{\mathrm{G} 5}=2$ and $\beta_{\mathrm{G} 6}=1$. This implies that G5 is more risk averse than G6, while other producers are risk-neutral.

- Case 4) this case is similar to Case 1 , but $\beta_{\mathrm{Q} 1}=2$ and $\beta_{\mathrm{Q} 2}=1$. In this case, both wind power producers are risk averse (with different weights), while all conventional producers are risk-neutral.

- Case 5) this case is similar to Case 1, but $\beta_{\mathrm{Q} 1}=$ $\beta_{\mathrm{Q} 2}=1$ and $\beta_{\mathrm{G} 5}=\beta_{\mathrm{G} 6}=10$. In this case, the two conventional producers G5 and G6 are more risk-averse than the two wind power producers.

In all Cases 1 to 5, the confidence levels of all producers, either conventional or renewable, are identical, i.e., $\alpha_{g}=$ $0.95, \forall g$ and $\alpha_{q}=0.95, \forall q$. The equilibrium results obtained by solving MCP (16)-(45) for different cases are provided in Table II. Rows 2 to 9 of this table refer to social marketclearing outcomes, while the next rows correspond to individual market-clearing outcomes for both wind power producers and some flexiable conventional producers, including G5, G6 and G7.

In Case 1, i.e., the risk-neutral case, the day-ahead and balancing markets are arbitraged, and therefore, the day-ahead and expected balancing prices are identical. Both wind power producers and each flexiable (reserve provider) conventional producer with high level of power schedules in day-ahead market are exposed to comparatively high risk. The reason for this is that their total generation levels in balancing stage change across different scenarios.

In Case 2, the conventional producer G5 is risk averse. Therefore, the expected profit of G5 decreases with respect to that in Case 1, while its CVaR increases. In this case, G5 is not scheduled in the day-ahead market. Instead of G5, the power schedule of the risk-neutral producer G6 is increased with respect to that in Case 1. Therefore, G6 is exposed to higher risk. This change in power schedules of conventional producers slightly increases the market prices, since the production cost of G6 is comparatively higher than that of G5. However, the day-ahead and expected balancing prices are still identical. Note also that the total schedules of conventional and wind power producers are not changed with respect to Case 1 .

In Case 3, both flexible producers G5 and G6 are risk averse, but with different weights. Therefore, their day-ahead schedules are comparatively lower than those in Cases 1 and 2. This leads to the lack of downward reserve sources in the balancing stages, and thus, the expected curtailed wind power increases. Thus, the expected profit of each wind power producer drops considerably, while that of each flexiable conventional producer increases. Note that the CVaR of each wind power producer is significantly low with respect to that in Cases 1 and 2. The market prices in Case 3 are slightly increased, however, the day-ahead and the expected balancing prices are still the same. In addition, the total schedules of conventional and wind power producers are identical to those in Cases 1 and 2.

In Case 4, both wind power producers are risk averse, whereas all conventional producers are risk-neutral. Therefore, compared to Case 1, the expected profit of each wind power 
producer decreases, while its CVaR significantly increases. The most important observation is that the wind power producers tend to be dispatched in balancing stage in order to reduce their own risk. Therefore, the total schedule of wind power producers in day-ahead market decreases from 758.4 MW in Cases 1-3 to 354.7 MW in Case 4, i.e., more wind power is available in balancing stage. In contrast, the total schedule of conventional producers in day-ahead market increases from 2234.1 MW in Cases 1-3 to 2637.8 MW in Case 4. Due to the risk considerations of both wind power producers, the dayahead market-clearing price is comparatively higher than the expected balancing price.

In Case 5, both wind power producers as well as flexiable producers G5 and G6 are risk averse (different weights). Therefore, all those producers tend to be less scheduled in day-ahead market. This increases the gap between day-ahead and expected balancing prices. Unlike producers G5 and G6, the most expensive but risk-neutral producer, i.e., G7, is fully dispatched in day-ahead market. The values for total expected wind power curtailment, expected system cost and total demand-side payment are highest in this case among all Cases 1 to 5 .

\section{CONCLUSiON}

This paper proposes a stochastic two-stage equilibrium model for perfectly competitive electricity markets (including day-ahead and balancing settlements) with risk-averse producers, and then evaluates the effects of risk aversion on market-clearing outcomes. This model is recast as a mixed complementarity problem.

The numerical results reveal that the risk-averse producers tend to be less scheduled in day-ahead market. This results in increasing the system cost and market-clearing prices. The most important conclusion of this work is that the day-ahead and the expected balancing prices may not be identical if all stochastic renewable producers are risk averse. The gap between those two prices even increases in a case in which the conventional reserve-provider producers are risk averse too.

\section{APPENDIX A}

The KKT conditions associated with optimization problem (1)-(8) for each conventional producer $g$ are given by (18)-(32) below:

$$
\begin{gathered}
C_{g}-\lambda^{\mathrm{DA}}+\bar{\mu}_{g}^{\mathrm{DA}}-\underline{\mu}_{g}^{\mathrm{DA}} \\
+\sum_{\omega}\left[\bar{\rho}_{g, \omega}^{\mathrm{B}}-\underline{\rho}_{g, \omega}^{\mathrm{B}}-\rho_{g, \omega}^{\mathrm{CVaR}}\left(\lambda^{\mathrm{DA}}-C_{g}\right)\right]=0 \quad \forall g \\
\phi_{\omega} C_{g}^{\mathrm{UP}}-\lambda_{\omega}^{\mathrm{B}}+\bar{\rho}_{g, \omega}^{\mathrm{B}}+\bar{\rho}_{g, \omega}^{\mathrm{UP}}-\underline{\rho}_{g, \omega}^{\mathrm{UP}} \\
-\rho_{g, \omega}^{\mathrm{CVaR}}\left(\frac{\lambda_{\omega}^{\mathrm{B}}}{\varphi_{\omega}}-C_{g}^{\mathrm{UP}}\right)=0 \quad \forall g, \forall \omega \\
\lambda_{\omega}^{\mathrm{B}}-\phi_{\omega} C_{g}^{\mathrm{DW}}+\underline{\rho}_{g, \omega}^{\mathrm{B}}+\bar{\rho}_{g, \omega}^{\mathrm{DW}}-\underline{\rho}_{g, \omega}^{\mathrm{DW}} \\
+\rho_{g, \omega}^{\mathrm{CVaR}}\left(\frac{\lambda_{\omega}^{\mathrm{B}}}{\varphi_{\omega}}-C_{g}^{\mathrm{DW}}\right)=0 \quad \forall g, \forall \omega
\end{gathered}
$$

$$
\begin{aligned}
& -\beta_{g}+\sum_{\omega} \rho_{g, \omega}^{\mathrm{CVaR}}=0 \quad \forall g \\
& \frac{\beta_{g} \phi_{\omega}}{1-\alpha_{g}}-\rho_{g, \omega}^{\eta}-\rho_{g, \omega}^{\mathrm{CVaR}}=0 \quad \forall g, \forall \omega \\
& 0 \leq p_{g}^{\mathrm{DA}} \perp \underline{\mu}_{g}^{\mathrm{DA}} \geq 0 \quad \forall g \\
& 0 \leq\left[P_{g}^{\max }-p_{g}^{\mathrm{DA}}\right] \perp \bar{\mu}_{g}^{\mathrm{DA}} \geq 0 \quad \forall g \\
& 0 \leq\left[P_{g}^{\max }-p_{g}^{\mathrm{DA}}-r_{g, \omega}^{\mathrm{UP}}\right] \perp \bar{\rho}_{g, \omega}^{\mathrm{B}} \geq 0 \quad \forall g, \forall \omega \\
& 0 \leq\left[p_{g}^{\mathrm{DA}}-r_{g, \omega}^{\mathrm{DW}}\right] \perp \underline{\rho}_{g, \omega}^{\mathrm{B}} \geq 0 \quad \forall g, \forall \omega \\
& 0 \leq r_{g, \omega}^{\mathrm{UP}} \perp \underline{\rho}_{g, \omega}^{\mathrm{UP}} \geq 0 \quad \forall g, \forall \omega \\
& 0 \leq\left[R_{g}^{\mathrm{UP}}-r_{g, \omega}^{\mathrm{UP}}\right] \perp \underline{\rho}_{g, \omega}^{\mathrm{UP}} \geq 0 \quad \forall g, \forall \omega \\
& 0 \leq r_{g, \omega}^{\mathrm{DW}} \perp \underline{\rho}_{g, \omega}^{\mathrm{DW}} \geq 0 \quad \forall g, \forall \omega \\
& 0 \leq\left[R_{g}^{\mathrm{DW}}-r_{g, \omega}^{\mathrm{DW}}\right] \perp \underline{\rho}_{g, \omega}^{\mathrm{DW}} \geq 0 \quad \forall g, \forall \omega \\
& 0 \leq \eta_{g, \omega} \perp \rho_{g, \omega}^{\eta} \geq 0 \quad \forall g, \forall \omega \\
& 0 \leq\left[\eta_{g, \omega}-\zeta_{g}+\left(p_{g}^{\mathrm{DA}}\left(\lambda^{\mathrm{DA}}-C_{g}\right)+\frac{\lambda_{\omega}^{\mathrm{B}}}{\varphi_{\omega}}\left(r_{g, \omega}^{\mathrm{UP}}-r_{g, \omega}^{\mathrm{DW}}\right)\right.\right. \\
& \left.\left.-C_{g}^{\mathrm{UP}} r_{g, \omega}^{\mathrm{UP}}+C_{g}^{\mathrm{DW}} r_{g, \omega}^{\mathrm{DW}}\right)\right] \perp \rho_{g, \omega}^{\mathrm{CVaR}} \geq 0 \quad \forall g, \forall \omega .
\end{aligned}
$$

\section{APPENDIX B}

The KKT conditions associated with optimization problem (9)-(13) for each renewable power producer $q$ are given by (33)-(42) below:

$$
\begin{aligned}
& -\lambda^{\mathrm{DA}}+\bar{\mu}_{q}^{\mathrm{DA}}-\underline{\mu}_{q}^{\mathrm{DA}} \\
& +\sum_{\omega}\left(\bar{\rho}_{q, \omega}^{\mathrm{B}}-\underline{\rho}_{q, \omega}^{\mathrm{B}}-\rho_{q, \omega}^{\mathrm{CVaR}} \lambda^{\mathrm{DA}}\right)=0 \quad \forall q \\
& -\lambda_{\omega}^{\mathrm{B}}+\bar{\rho}_{q, \omega}^{\mathrm{B}}-\underline{\rho}_{q, \omega}^{\mathrm{B}}-\rho_{q, \omega}^{\mathrm{CVaR}} \frac{\lambda_{\omega}^{\mathrm{B}}}{\varphi_{\omega}}=0 \quad \forall q, \forall \omega \\
& -\beta_{q}+\sum_{\omega} \rho_{q, \omega}^{\mathrm{CVaR}}=0 \quad \forall q \\
& \frac{\beta_{q} \phi_{\omega}}{1-\alpha_{q}}-\rho_{q, \omega}^{\eta}-\rho_{q, \omega}^{\mathrm{CVaR}}=0 \quad \forall q, \forall \omega \\
& 0 \leq w_{q}^{\mathrm{DA}} \perp \underline{\mu}_{q}^{\mathrm{DA}} \geq 0 \quad \forall q \\
& 0 \leq\left[W_{q}^{\mathrm{max}}-w_{q}^{\mathrm{DA}}\right] \perp \bar{\mu}_{q}^{\mathrm{DA}} \geq 0 \quad \forall q \\
& 0 \leq\left[w_{q}^{\mathrm{DA}}+w_{q, \omega}^{\mathrm{B}} \perp \underline{\rho}_{q, \omega}^{\mathrm{B}} \geq 0 \quad \forall q, \forall \omega\right. \\
& 0 \leq\left[W_{q, \omega}-w_{q}^{\mathrm{DA}}-w_{q, \omega}^{\mathrm{B}} \perp \perp \bar{\rho}_{q, \omega}^{\mathrm{B}} \geq 0 \quad \forall q, \forall \omega\right.
\end{aligned}
$$




$$
\begin{aligned}
0 \leq \eta_{q, \omega} \perp \rho_{q, \omega}^{\eta} \geq 0 \quad \forall q, \forall \omega \\
0 \leq\left[\eta_{q, \omega}-\zeta_{q}+\left(\begin{array}{c}
\left.\left.w_{q}^{\mathrm{DA}} \lambda^{\mathrm{DA}}+\frac{\lambda_{\omega}^{\mathrm{B}}}{\varphi_{\omega}} w_{q, \omega}^{\mathrm{B}}\right)\right] \\
\quad \perp \rho_{q, \omega}^{\mathrm{CVaR}} \geq 0 \quad \forall q, \forall \omega .
\end{array}\right.\right.
\end{aligned}
$$

\section{APPENDIX C}

The KKT conditions associated with optimization problem (14)-(15) for each load $l$ are given by (43)-(45) below:

$$
\begin{aligned}
& \phi_{\omega} V_{d}-\lambda_{\omega}^{\mathrm{B}}+\bar{\rho}_{l, \omega}^{\mathrm{cur}}-\underline{\rho}_{l, \omega}^{\mathrm{cur}}=0 \quad \forall l, \forall \omega \\
& 0 \leq p_{l, \omega}^{\mathrm{cur}} \perp \underline{\rho}_{l, \omega}^{\mathrm{cur}} \geq 0 \quad \forall l, \forall \omega \\
& 0 \leq\left[P_{l}-p_{l, \omega}^{\mathrm{cur}}\right] \perp \bar{\rho}_{l, \omega}^{\mathrm{cur}} \geq 0 \quad \forall l, \forall \omega .
\end{aligned}
$$

\section{REFERENCES}

[1] G. Pritchard, G. Zakeri, and A. Philpott, "A single-settlement, energyonly electric power market or unpredictable and intermittent participants," Oper. Res., vol. 58, no. 4, pp. 1210-1219, Jul. -Aug. 2010.

[2] S. Wong and J. D. Fuller, "Pricing energy and reserves using stochastic optimization in an alternative electricity market," IEEE Trans. Power Syst., vol. 22, no. 2, pp. 631-638, May 2007.

[3] J. M. Morales, A. J. Conejo, K. Liu, and J. Zhong, "Pricing electricity in pools with wind producers," IEEE Trans. Power Syst., vol. 27, no.3, pp. 1366-1376, Aug. 2012.

[4] J. M. Morales, M. Zugno, S. Pineda, and P. Pinson, "Electricity market clearing with improved scheduling of stochastic production," Eur. J. Oper. Res., vol. 235, no. 3, pp. 765-774, Jun. 2014.

[5] S. Martín, Y. Smeers, and J. A. Aguado, "A stochastic two settlement equilibrium model for electricity markets with wind generation," IEEE Trans. Power Syst., vol. 30, no. 1, pp. 233-245, Jan. 2015.

[6] Y. Zhang and G. B. Giannakis, "Distributed stochastic market clearing with high-penetration wind power," IEEE Trans. Power Syst., vol. 31, no. 2, pp. 895-906, Mar. 2016.

[7] N. Zhang, C. Kang, Q. Xia, Y. Ding, Y. Huang, R. Sun, J. Huang, and J. Bai, "A convex model of risk-based unit commitment for day-ahead market clearing considering wind power uncertainty," IEEE Trans. Power Syst., vol. 30, no. 3, pp. 1582-1592, May 2015.

[8] R. T. Rockafellar and S. Uryasev, "Optimization of conditional value-atrisk," J. Risk, vol. 2, pp. 21-41, 2000.

[9] B. F. Hobbs, "Linear complementarity models of Nash-Cournot competition in bilateral and POOLCO power markets," IEEE Trans. Power Syst., vol. 16, no. 2, pp. 194-202, May 2001.

[10] Reliability System Task Force, "The IEEE reliability test system1996: a report prepared by the reliability test system task force of the application of probability methods subcommittee" IEEE Trans. Power Syst., vol. 14, no. 3, pp. 1010-1020, Aug. 1999. 